\title{
Can guidelines improve referral to elective surgical specialties for adults? A systematic review
}

\author{
A Clarke, ${ }^{1}$ N Blundell, ${ }^{2}$ I Forde, ${ }^{3}$ N Musila, ${ }^{4}$ D Spitzer, ${ }^{5}$ S Naqvi, ${ }^{1}$ J Browne ${ }^{4}$
}

\begin{abstract}
${ }^{1}$ Health Sciences Research Institute, University of Warwick, Coventry, USA

${ }^{2}$ Research in Practice for Adults, Blacklers, Dartington, Totnes, Devon, UK

${ }^{3}$ Department of Epidemiology and Public Health, University College, London, UK

${ }^{4}$ Health Services Research Unit, London School of Hygiene and Tropical Medicine, London, UK ${ }^{5}$ Centre for Health Sciences, Barts \& the London School of Medicine \& Dentistry, London, UK
\end{abstract}

\section{Correspondence to}

Aileen Clarke, Warwick Medical School, University of Warwick, Health Sciences Research Institute, Coventry CV4 7AL, UK; aileen.clarke@warwick.ac.uk

Accepted 5 March 2009

\section{(D) ULOCKID}

This paper is freely available online under the BMJ Journals unlocked scheme, see http:// qshc.bmi.com/site/about/ unlocked.xhtml

\section{ABSTRACT}

Aim To assess effectiveness of guidelines for referral for elective surgical assessment.

Method Systematic review with descriptive synthesis. Data sources Medline, EMBASE, CINAHL and Cochrane database up to 2008. Hand searches of journals and websites.

Selection of studies Studies evaluated guidelines for referral from primary to secondary care, for elective surgical assessment for adults.

Outcome measures Appropriateness of referral (usually measured as guideline compliance) including clinical appropriateness, appropriateness of destination and of pre-referral management (eg, diagnostic investigations), general practitioner knowledge of referral appropriateness, referral rates, health outcomes and costs.

Results 24 eligible studies ( 5 randomised control trials, 6 cohort, 13 case series) included guidelines from UK, Europe, Canada and the USA for referral for musculoskeletal, urological, ENT, gynaecology, general surgical and ophthalmological conditions. Interventions varied from complex ("one-stop shops") to simple guidelines. Four randomized control trials reported increases in appropriateness of pre-referral care (diagnostic investigations and treatment). No evidence was found for effects on practitioner knowledge. Mixed evidence was reported on rates of referral and costs (rates and costs increased, decreased or stayed the same). Two studies reported on health outcomes finding no change.

Conclusions Guidelines for elective surgical referral can improve appropriateness of care by improving prereferral investigation and treatment, but there is no strong evidence in favour of other beneficial effects.

In many health systems, the general practitioner or primary care gatekeeper role is considered a vital component of demand management, restricting access to secondary care based on identified need, thus restraining healthcare costs. However, evidence suggests substantial variation in referral rates from primary to secondary care, indicating that access to surgery is not equitable. ${ }^{1-3}$ In the UK, guidance for the referral of common conditions to surgical specialties has been provided, with the potential to increase the equity of referral, while maintaining a "gatekeeper role". This guidance has not as yet been rigorously implemented or evaluated. ${ }^{4}$ Two reviews have investigated interventions for referral from primary to secondary care in general. ${ }^{5}{ }^{6}$ Faulkner et al ${ }^{6}$ reported little impact of referral guidelines on rates of referral or health outcomes. Grimshaw et al found that passively disseminated guidelines had less impact than structured referral sheets.
The standard definition of clinical guidelines is "systematically developed statements to assist practitioner and patient decisions about appropriate healthcare for specific clinical circumstances". 7 Although their principal intended benefit is to improve the quality of care received by patients, several other benefits to patients, clinicians and healthcare systems have been suggested, including improving consistency and equity of care, empowering patient participation and influencing public policy-for example, through highlighting neglected health problems. ${ }^{8}$

Nevertheless, the evidence that guidelines alone are effective at changing clinician behaviour is limited..$^{9-12}$ In light of concern about increasing referrals to secondary care, the introduction of "patient choice" and increasing plurality of elective surgical service providers, the UK Department of Health commissioned this work to answer the following questions: "do guidelines for referral by primary care practitioners for adults for elective surgical assessment improve appropriateness of referral or health outcomes? What is the effect of referral guidelines on healthcare costs?"

Referral guidelines were defined as "a set of rules, an algorithm or a protocol which gives guidance on the circumstances in which a formal elective request is made from primary care on behalf of a patient, for treatment or consultation by a surgical specialist". (A surgical specialist was defined as a medical practitioner who has undertaken a recognised training to be able to undertake elective, non-urgent surgery). We aimed to investigate the key characteristics of guidelines including format, length, development method and any prescribed or implicit associated implementation strategies.

\section{METHODS}

The search strategy was developed based on extensive literature scoping exercise, and advice from and clinical, health information and health management experts (including those involved in the Cochrane Collaboration Effective Practice an Organisation of Care Group, practising NHS managers and clinicians, and primary care practitioners/general practitioners with an interest in elective surgical referral). To maximise sensitivity, both text words and subject headings (MeSH terms) were included. Four topic areas ("primary healthcare or family practice", "practice guidelines or algorithms", "referral and consultation", and "surgical procedures or "surgery") were all combined using the "and" operator. The final search strategy is included in Box 1.

Searches were conducted in Medline, EMBASE, CINAHL and the Cochrane Database from 


\section{Box 1 Search strategy}

Family Practice (MeSH) OR Primary Health Care (MeSH) OR Physicians, Family (MeSH) OR Primary Care OR "managed care" OR general practi* OR GP OR GPs OR family doctor* OR family physician* $\mathrm{OR}$ generalist*

AND

Referral and Consultation (MeSH) OR refer OR referr* $\mathrm{OR}$ ((recommend* OR request*) AND (Surgical Procedures, Operative (MeSH) OR Surgery (MeSH) OR surgeon* OR surgery (Text Word) OR surgical (Text Word) OR operate (Text Word) OR operative (Text Word) OR operation (Text Word) OR operations (Text Word))!

AND

Practice Guidelines (MeSH) OR Algorithms (MeSH) OR guideline* $O R$ guidance $O R$ tool OR tools OR "algorithm" OR "algorithms" OR protocol OR protocols OR pathway* OR "care standards" OR "treatment standards" OR "preferred practice patterns" OR "decision tree" OR "decision trees" OR "decision aid" OR "decision aids" OR "decision modelling" OR "decision modelling".

*denotes generic ending.

inception to February 2008. Hand searches of relevant selected journals (British Journal of General Practice, Journal of Health Services Research \& Policy, Quality and Safety in Health Care) and websites were undertaken, and these were supplemented with examination of reference lists and contact with experts. Titles and abstracts were inspected and full texts of potentially relevant publications were each assessed for inclusion by two reviewers. Table 1 shows inclusion and exclusion criteria.

A data extraction form was designed, based on guidance from the Scottish Intercollegiate Guidelines Network ${ }^{13}$ and the NHS Centre for Reviews and Dissemination guidance. ${ }^{14}$ Data were extracted by two reviewers with discrepancies resolved through discussion. Studies were appraised using recommended critical appraisal tools and two authors working together allocated a Scottish Intercollegiate Guidelines Network quality score.

Studies were not excluded based on methods or methodological quality. Outcomes of interest assessed were general practitioners' knowledge of appropriateness of referral, actual appropriateness of referral (including appropriateness of diagnostic tests, effects of guidelines on referral rates and on waiting times-often referred to as guidelines compliance in studies), health outcomes and costs. Because of the nature of the topic and the heterogeneity of the studies (interventions, patient groups and outcomes measures used), quantitative summary estimates of effect were not calculated. Data are therefore synthesised descriptively.

\section{RESULTS}

Initial searches yielded 9398 papers after duplicates were removed. Nine thousand two hundred and seventy were excluded by reading title and abstract, with uncertainty resolved by discussion between two reviewers. Full texts of 128 papers were retrieved and examined by two reviewers. Discrepancies were resolved by discussion with a third reviewer and 24 papers were identified for inclusion. Figure 1 gives a flow diagram and table 2 summarises the characteristics of the included studies. Publication dates ranged from 1993 to 2007. Ten of the studies were conducted in the UK and the remainder in North America and Europe. Guidelines were variably developed for local, national or international use.

\section{Study design}

Eleven controlled designs included data on $>9000$ referrals. Five studies were randomised controlled trials. Four used cluster randomisation and one used patient-level randomisation. ${ }^{15-19}$ There were six cohort designs of which four used historical controls,$^{20-23}$ one used concurrent controls ${ }^{24}$ and one was of mixed design, using a combination of historic and concurrent non-randomised controls. ${ }^{25}$ Thirteen studies, covering $>12000$ referrals, used an uncontrolled case series design. ${ }^{12} 26-37$

\section{Clinical area of interest}

Guidelines covered elective surgical referral for patients to orthopaedic surgeons (low back pain and other orthopaedic conditions), urologists (lower urinary tract symptoms and microscopic haematuria), otolaryngology (ENT), gynaecologists and ophthalmologists. Some guidelines explicitly covered more than one clinical area of interest, particularly those for referral to general surgeons (eg, for hernia repair and other minor surgery).

\section{Interventions}

Only three studies provided a simple evaluation of referral guidelines. All these were descriptive designs with two reporting no effects and one reporting benefits. The other studies investigated guidelines that had an associated strategy aimed at enhancing dissemination. These strategies included the following:

1. overall disease management guidelines, which included explicit referral guidelines (13 studies)-for example, a structured management sheet. Some of these included an accompanying educational package for referrers. Of the seven controlled studies that reported on this type of intervention, five reported positive benefits.

2. referral guidelines as part of services explicitly changing and simplifying the patient care pathway usually with accompanying greater integration of primary and secondary care (often referred to as "one-stop shop services") (six studies)-for example, guideline-based direct access to surgical waiting lists. Both controlled studies reporting on one-stop shop services reported benefits.

3. guideline-based referral triage (which included referral to a central secondary care referral management centre with redistribution to appropriate providers) (two studies). One of the latter was a management guideline where referrers had to obtain telephone-based previous authorisation for referral. The single controlled study of this intervention reported mixed effects.

\section{Guideline development process}

Methods of guideline development used in various combinations were consensus development panels, multidisciplinary panels, specialist experience, general practitioner consultation and literature review. None of the studies mentioned patient input either at the individual or the representative level. Nineteen of the studies evaluated locally or regionally developed guidelines, and of these, 18 reported benefits or mixed effects and one reported no benefit. Two descriptive and three controlled studies described local evaluations of nationally or internationally developed guidelines, of which only two reported overall positive results and three reported no benefits.

Table 3 shows study characteristics for descriptive and controlled designs. 
Table 1 Inclusion and exclusion criteria

\begin{tabular}{|c|c|c|}
\hline Selection criteria & Inclusion criteria & Exclusion criteria \\
\hline \multirow[t]{4}{*}{ Population patients } & - Adults (aged 16 years +) & - Children (aged <16 years) \\
\hline & - With a non-urgent condition & $\begin{array}{l}\text { With an urgent condition requiring emergency or urgent } \\
\text { referral } \\
\text { "Red flag" back symptoms } \\
\text { - Any evaluation of cancer-eg, fitting UK "2-week rule" } \\
\text { guidelines }\end{array}$ \\
\hline & $\begin{array}{l}\text { - Guideline consultation } \\
\text { - In primary care by a PCP/GP }\end{array}$ & $\begin{array}{l}\text { - Guideline consultation } \\
\text { - In primary dental care by general dental practitioner } \\
\text { - Undertaken by optician/optometrist } \\
\text { - In Accident and Emergency department } \\
\text { - In secondary care }\end{array}$ \\
\hline & $\begin{array}{l}\text { Condition should be amenable to surgical intervention if } \\
\text { severe enough }\end{array}$ & - Condition not amenable to surgical intervention \\
\hline
\end{tabular}

severe enough

-Do include infertility if referral is from primary to secondary care; back pain if surgery is an option; glaucoma if referral is from primary to secondary care and surgery is an option; breast symptoms if non-urgent component

Population practitioners $\quad$ Referring practitioner is GP or PCP

- Receiving practitioner is a surgeon or practitioner in surgical specialty in secondary care

Interventions

Outcomes

Study designs
- Any guideline(s) or set of rules or protocol that assists PCPs with a decision of whether or not to refer patients to a surgeon or surgical specialty in secondary care for further advice, consultation or treatment

- Referral for endoscopy or other diagnostic tests if referral is for management of symptoms, not just for investigation

Any assessments of

- Appropriateness of referral

- Change in GP/PCP knowledge

- Change in disease status/change in health status/quality of life

- Costs

- No study design excluded

- Evaluation of a referral guideline OR study measuring compliance with specific named guideline (comparison of actual practice with guideline)

- Publication must be research based with original data Referring practitioner is not a PCP (eg, in referral to tertiary or
high dependency care facilities)
- Receiving practitioner is a someone other than a surgeon or
practitioner in a surgical specialty (eg, social services,
complementary therapies, district nursing, etc)
- There is no identifiable (repeatable, written) set of rules that
could be generalised to GP/PCPs in-eg, another geographic
area
Referral is for diagnostic tests only
Back pain if referral is for physiotherapy or imaging
Outcomes identified do not fall into the five identified
categories of outcome
- No study design excluded
No evaluation or comparison with guideline
presented clear, identifiable guideline(s) named

$\mathrm{GP}$, general practitioner; $\mathrm{PCP}$, primary care practitioner

\section{Study findings - descriptive designs}

Quality assessment

Thirteen of the studies used a descriptive (mostly case series) design. For all, outcome assessment was undertaken by unblinded assessors (usually the authors who had designed the guidelines). One study used a cross-sectional design and one was a modelling study. None of these studies used comparators of controls. These studies all have a high risk of bias and confounding, and their quantitative results are open to misinterpretation. However, they are useful for delineating the area (eg, the range of outcomes considered) and for demonstrating the feasibility of use of referral guidelines in practice.

\section{Summary of themes from descriptive designs}

Eight of the thirteen studies reported guidelines as beneficial for patient care. Outcomes investigated included appropriateness of referral (often assessed as compliance with guidelines), reduction in waiting times or costs, and change in referral rates. None of the descriptive studies reported on health outcomes.

\section{Study findings - controlled designs}

Quality assessment

Eleven studies used controlled designs. Six studies were cohort designs. In these studies, subjects were not randomly selected and outcome assessors were not blinded to intervention status.
Almost all of these studies have a high risk of confounding and bias, and there is a significant risk that the relationships found are not causal. One ${ }^{24}$ with concurrent (non-randomised) controls was carefully conducted to minimise bias. Of the five randomized control trials, four were cluster randomised and one was randomised at the individual level. All described clear eligibility criteria, comparability of baseline measures and described an intention-to-treat analysis. Only two ${ }^{15} 18$ described an adequate approach to sequence generation and allocation concealment and were therefore considered to have a low risk of bias.

\section{General practitioners' knowledge of appropriateness of referral} None of the controlled studies reported on this outcome.

\section{Effect of referral guidelines on appropriateness of referral}

Two cohort studies reported overall improvement in referral appropriateness as a result of guideline implementation. One study in otorhinolaryngology $(\mathrm{ENT})^{21}$ reported a statistically significant improvement in the proportion of appropriate referrals (assessed by an independent specialist) after the intervention.

Four studies reported on the impact of referral guidelines on the appropriateness of investigations carried out by general practitioners before making a referral. ${ }^{15} \quad 161923$ One cluster randomised trial, evaluating a "one-stop service" for urological conditions, reported a significant improvement of 0.5 in 


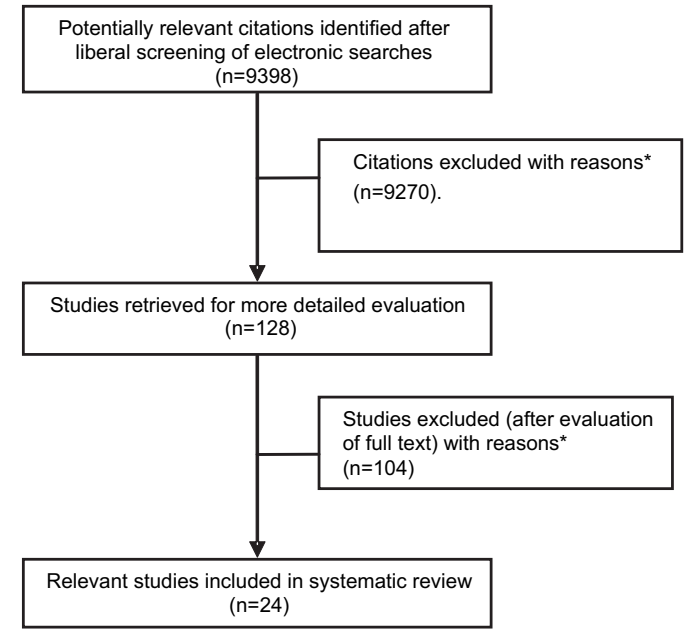

*Unrelated to referral or not referral from primary to secondary care. Not elective surgical. Not related to guidelines. Not an evaluation. Duplicate publication

(See inclusion/exclusion criteria. See Box 2 for further details.)

Figure 1 Flow (QUORUM) diagram. Study identification and selection.

a "compliance score" (range 0-5, based on the number of guideline-recommended investigations carried out before referral). ${ }^{15}$ Both randomized control trials of use of a guideline plus structured management sheet for patients with infertility ${ }^{16}$

19 reported significant improvements in the appropriateness of diagnostic investigations undertaken before referral and in the patient histories recorded ( $72 \%$ vs $41 \%(n=100)$ correct assessment of day 21 progesterone levels $(\mathrm{p}<0.001))^{19}$ (mean number of relevant tests 2.81 vs 2.50 ; odds ratio $1.32 ; 95 \%$ CI 1.00 to 1.75 ; $\mathrm{p}=0.025) .{ }^{16}$ A cohort study with historical controls of a management guideline for patients with lower urinary tract symptoms also reported an increase in appropriate use of investigations. (Digital rectal examination (recommended in the guideline) increased from $32 \%$ to $41.1 \%(n=1203, p<0.001)$ and transrectal and suprapubic ultrasonography (not recommended in the guideline) decreased from $33 \%$ to $23 \%$ and $53 \%$ to $44 \%$ $(p<0.001)$, respectively. $)^{23}$

All the controlled studies that provided evidence on this variable reported that referral guidelines improved the appropriateness of diagnostic evaluations carried out by general practitioners before referral.

We can draw no overall conclusions about the impact of guidelines on the likelihood of referral because studies used a variety of measures (both rates and numbers of referrals). Four studies reported a reduction in crude numbers, three reported no change and one suggested that guidelines would increase the

Table 2 Levels of evidence

$1++\begin{aligned} & \text { High quality meta-analyses, systematic reviews of RCTs or RCTs with a very } \\ & \text { low risk of bias } \\ & 1+ \\ & \text { Well-conducted meta-analyses, systematic reviews or RCTs with a low risk of } \\ & \text { bias } \\ & 1-\quad \text { Meta-analyses, systematic reviews or RCTs with a high risk of bias } \\ & 2++ \text { High-quality systematic reviews of case control or cohort or studies, high- } \\ & \text { quality case control or cohort studies with a very low risk of confounding or } \\ & \text { bias, and a high probability that the relationship is causal }\end{aligned}$
$2+\quad \begin{aligned} & \text { Well-conducted case control or cohort studies with a low risk of confounding or } \\ & \text { bias and a moderate probability that the relationship is causal }\end{aligned}$
$\begin{aligned} & \text { Case control or cohort studies with a high risk of confounding or bias and } \\ & \text { a significant risk that the relationship is not causal }\end{aligned}$
$\begin{aligned} & \text { Non-analytic studies-eg, case reports, case series } \\ & 4\end{aligned}$
Expert opinion

RCT, randomized control trial. referral rate. Two well-designed randomised trials reported no change in rates and one reported a reduction in referral rates in intervention as compared to control groups.

Five studies reported the impact of interventions on waiting times. A variety of start and end points were used. Periods of time measured were time from referral to first specialist consultation, time from referral to surgery and time from first general practitioner visit to final diagnosis; and different methods of measuring waiting time were used (mean waiting time and percentage of eligible patients seen by a specialist within a given time from the date of referral).

Three studies reported reductions in waiting times; one reported mixed results and one reported no change. As an example, the cohort study examining impact of a direct access to surgical waiting list intervention with referral guideline reported that for patients with hernia, the median wait for intervention patients was reduced by 91 days compared to the median wait for control patients $(\mathrm{p}<0.0001){ }^{25}$

\section{Impact of referral guidelines on cost and health outcomes}

a. Cost No studies reported a formal economic evaluation. One cohort study with historical controls of a management guideline for patients with lower urinary tract symptoms reported a reduction in costs of between $£ 1.45$ and $£ 49.54$ per patient (at 2000 prices). ${ }^{24}$ In contrast, a cluster randomised trial of a guideline plus structured management sheet for infertility reported a non-significantly higher median cost per referral in intervention practices (increase of 18\%) £215-251 (at 1998 prices). ${ }^{16}$

b. Health outcomes Two studies reported explicitly on the impact of guidelines on patient health outcomes using the Short Form Health Survey (SF-36), a set of generic quality-oflife measures. Both reported no difference between intervention and control groups.

\section{DISCUSSION}

\section{Summary of results}

We undertook a systematic review of guidelines for elective referral to surgical specialist. We identified 24 studies, of which only five used randomised designs, although a further six included data from comparison or control groups. Studies were based on data from large numbers of patients, from a number of different countries and covered a range of conditions. Most of the studies we found $(21 / 24)$ reported evaluations of complex interventions that included an associated implementation or management plan. Of these, overall disease management guidelines and one-stop shop arrangements appear to confer benefits, although the absolute number of studies of each is small.

Studies reached contradictory conclusions regarding the effect of guidelines on general practitioners' knowledge and awareness of appropriateness of referral for the conditions under investigation. Controlled studies measuring compliance with guideline referral criteria reported improvements. All studies that assessed appropriateness of diagnostic evaluations carried out before referral after use of a referral guideline reported improvements.

We could draw no overall conclusions about the impact of referral guidelines on rates of referral based on the studies identified. Although many studies reported data on reduction of overall waiting times to surgery, attribution to referral guidelines of the effects found is problematic. No formal evaluations of costs and benefits of referral guidelines were found. Only two studies assessed patient outcomes, with no effects found. 


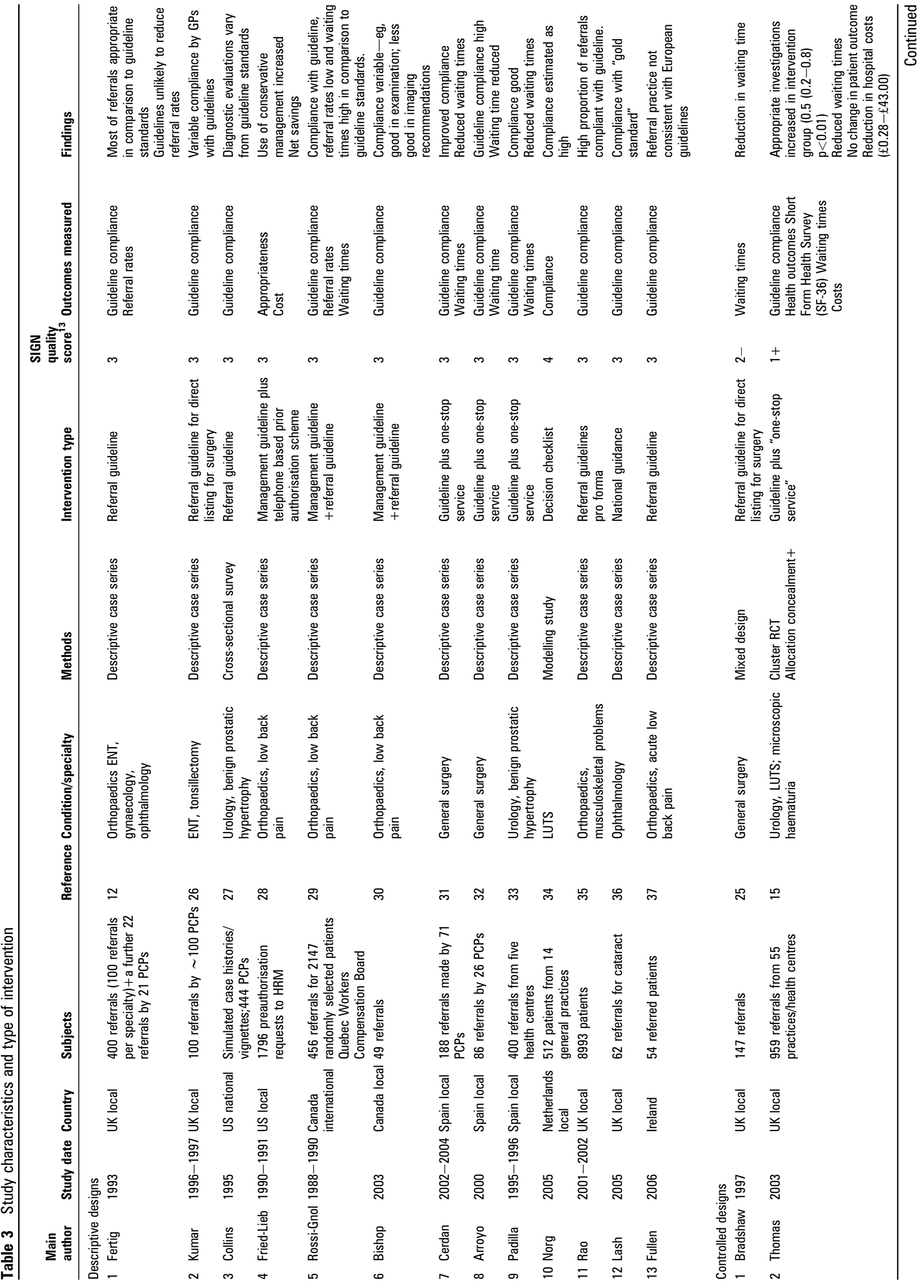

일

$\stackrel{\mathscr{N}}{\exists}$ 


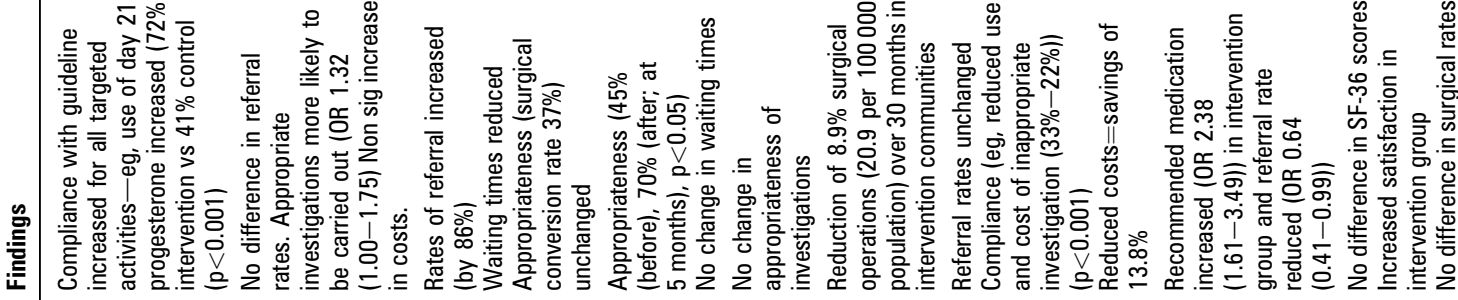
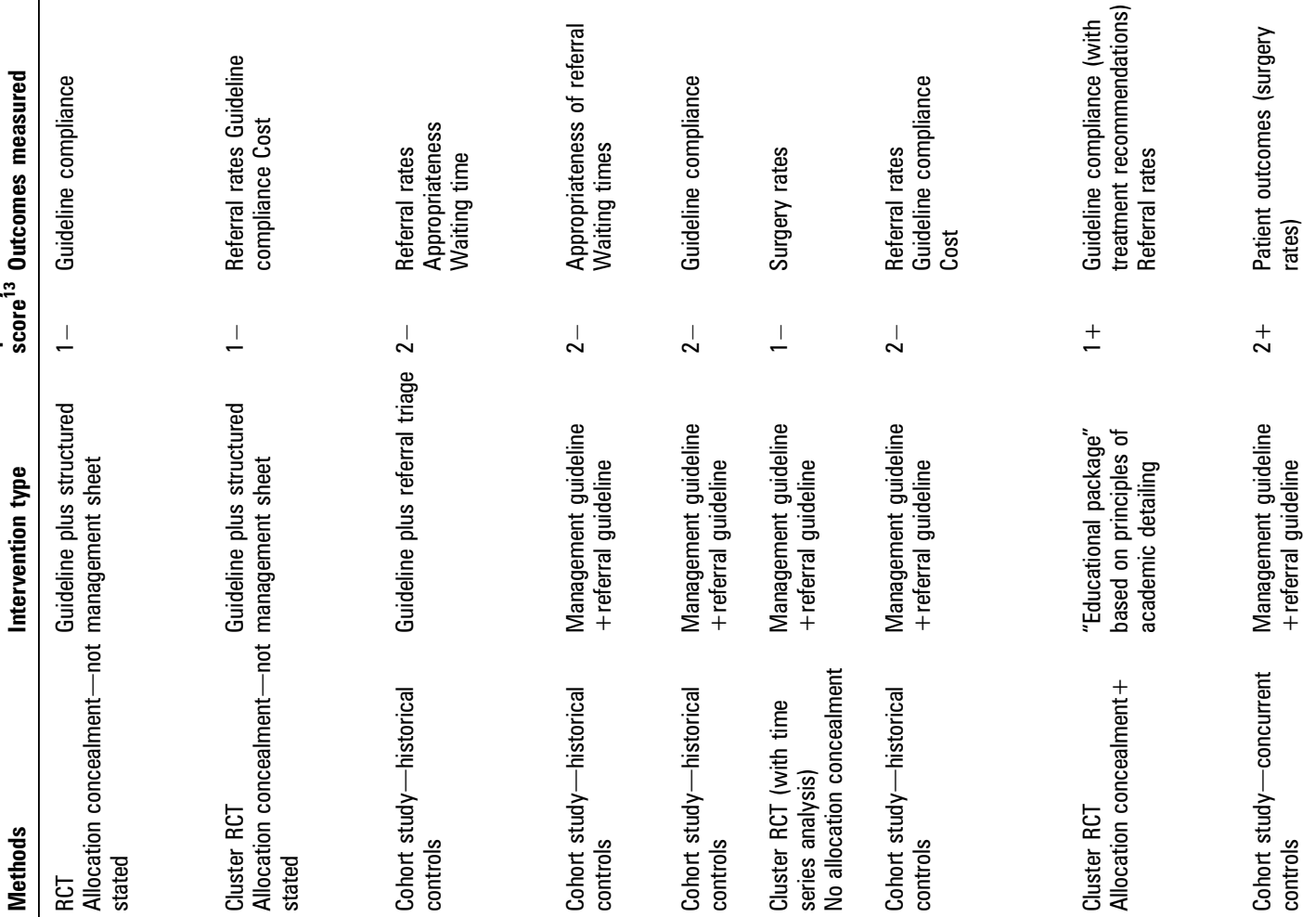

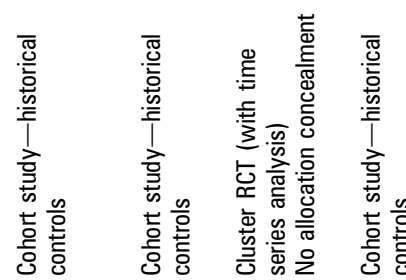
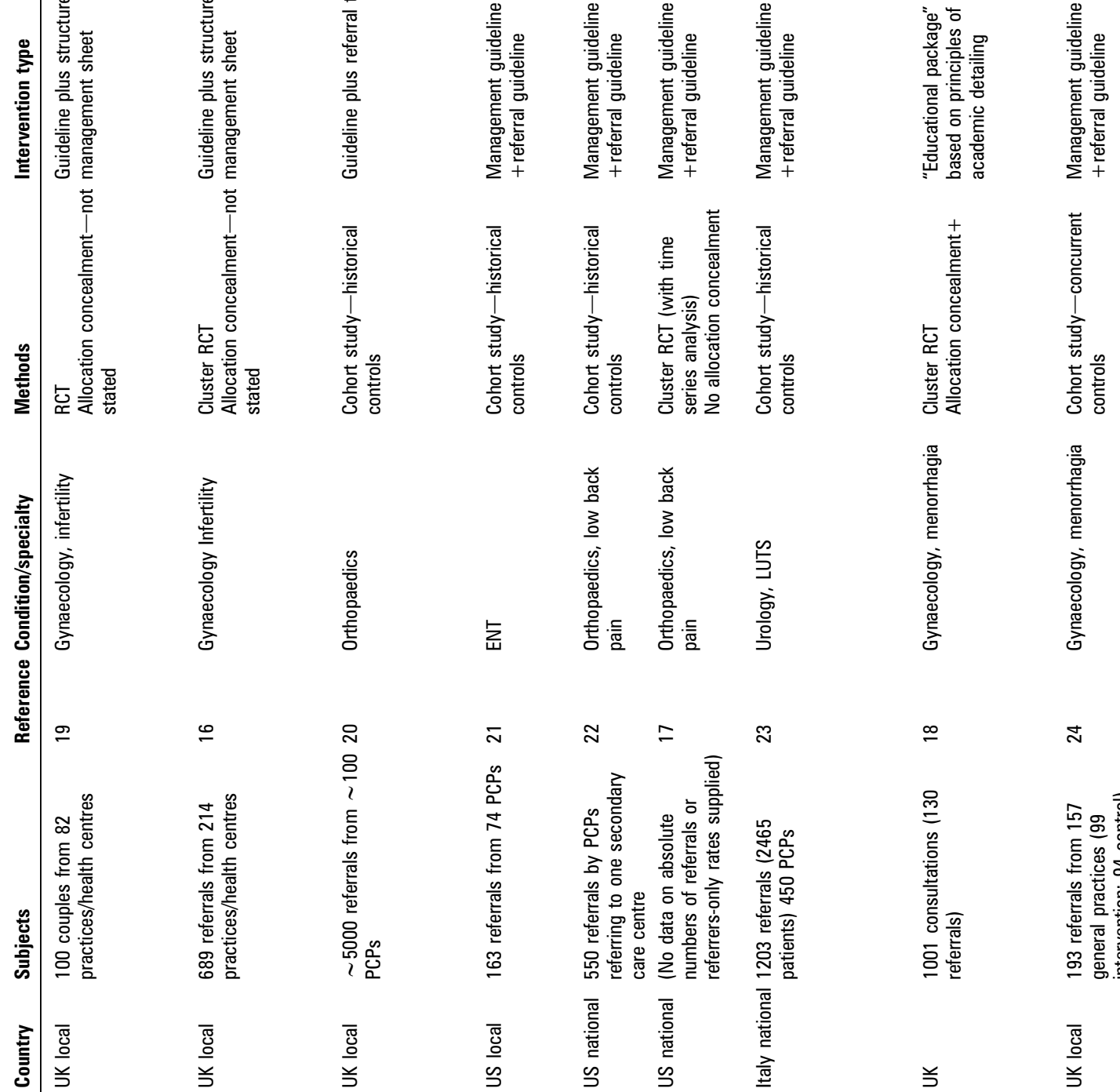

증

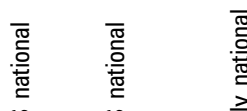

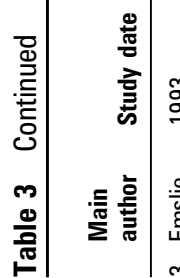

㜽

商

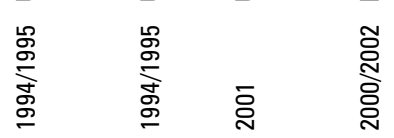

兽

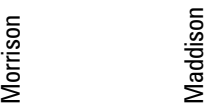




\section{Strengths and weaknesses}

In this review we concentrated on guidelines for referral to surgical specialties by general practitioners or primary care practitioners for adults with non-urgent conditions. Strengths of the review are that unlike the two previously published reviews, ${ }^{5}$ we focused solely on referral guidelines for elective surgical assessment and we did not restrict our searches to randomised controlled trials. Extensive work went into developing a search strategy, which accurately covered the full range of terms used to describe the concept of "referral." As a result we have identified a number of relevant studies internationally in addition to those covered in existing reviews. We did not estimate publication bias systematically, although it is noticeable that authors evaluating guidelines that they had produced themselves or that had been produced locally were more likely to be evaluated positively. Five of the included studies covered national guidelines produced from "elsewhere" and disseminated passively, and these tended to be evaluated less positively. Generalisability is an issue because all the included studies we found were undertaken in high-income countries and, of these, 18 of 24 were from English-speaking countries.

We did not synthesise results to produce overall quantitative conclusions on the benefits of referral guidelines because of the disparities in interventions, definitions, measures and outcomes used in the current body of research. Summary estimates of effect (if indeed they were possible to derive) would at this stage be misleading. Instead, we undertook a descriptive synthesis of the findings, ${ }^{38}$ taking account of the relative weight of evidence using a recognised strength of evidence tool. ${ }^{13}$

\section{Implications for policy, practice and research}

Guidelines for referral for elective surgical assessment by primary care practitioners appear to improve appropriateness of referral by improving appropriateness of pre-referral diagnostic investigation. However, there is no consistent evidence for effects on other measures of appropriateness of referral or on health outcomes. There is conflicting evidence on the effect of these guidelines on costs. Notwithstanding these problems, guideline production has consistently been encouraged as one of the means to implement research findings into clinical practice. And elective surgical referral is a well-defined area where guidelines should be of value. Our findings suggest that referral guidelines on their own are unlikely to improve referrals especially those where dissemination is passive, and the findings of this review confirms previous reports.

However, our findings about different methods of active implementation are interesting. It is clear that those wishing to adopt referral guidelines need to adopt and tailor an associated implementation strategy, which is most suitable to their local circumstances.

All but a few of the studies of effectiveness of guidelines for elective surgical referral that we found were not of high methodological quality. High-quality evaluations of referral guidelines are still clearly needed. Other issues for further research include:

- investigation into the complex management intervention(s) that best support referral guideline use and compliance

- teasing out of the effects of bias because of local championing of local guidelines and the legitimate benefits of a local development approach

Guidelines may only ever be a part of the story, and there may also be a need for some "back to the drawing board" research into the best methods for ensuring that a rapidly developing evidence base can be incorporated into these important, nodal "gateway" decisions made by primary care practitioners in healthcare.

Funding The study was funded by the NIHR SDO research and development programme (grant SDO/08/1310/072). The study funders had no role in study design; in the collection, analysis and interpretation of data; in the writing of the report; and in the decision to submit the article for publication. The researchers were independent from the funders.

Competing interests Declared. AC, NM and JB are involved in phase II of this work which involves development and evaluation of referral guidelines for elective surgical assessment in orthopaedics and urology.

Provenance and peer review Not commissioned; externally peer reviewed.

\section{REFERENCES}

1. O'Donnell C. Variation in GP referral rates: what can we learn from the literature? Fam Pract 2000;17:462-71.

2. Reynolds G, Chitnis J, Roland M. General practitioner outpatient referrals: do good doctors refer more patients to hospital? BMJ 1991;302:1250-2.

3. Wilkin D, Smith A. Variation in general practitioners' referral rates to consultants. J $R$ Coll Gen Pract 1987; 37:350-3.

4. National Institute of Clinical Excellence. (NICE) Referral advice: a guide to appropriate referral from general to specialist services. 2001. ISBN: 1-84257144-3 http://www.nice.org.uk/media/A8F/DC/Referraladvice.pdf laccessed 4 Jan 2010)

5. Grimshaw JM, Winkens RAG, Shirran E, et al. Interventions to improve outpatient referrals from primary care to secondary care. Cochrane Database Syst Rev $2005 \mathrm{Ju}$ 20;3:CD005471

6. Faulkner A, Mills N, Bainton, et al. A systematic review of the effect of primary carebased service innovations on quality and patterns of referral to specialist secondary care. Br J Gen Pract 2003;53:878-84.

7. Field M, Lohr K. Clinical practice guidelines: directions for a new program Washington, DC. National Academy Press; 1990.

8. Woolf S, Grol R, Hutchinson A, et al. Clinical guidelines: potential benefits, limitations, and harms of clinical guidelines. BMJ 1999;318:527-30.

9. Gabbay J, le May A. Evidence based guidelines or collectively constructed "mindlines?" Ethnographic study of knowledge management in primary care. BMJ 2004;329:1013

10. Worrall G, Chaulk P, Freake D. The effects of clinical practice guidelines on patient outcomes in primary care: a systematic review. CMAJ 1997; 156:1705-12.

11. Grimshaw J, Shirran L, Thomas R, et al. Changing provider behavior: an overview of systematic reviews of interventions. Med Care 2001;39:॥2-45.

12. Fertig A, Roland $M$, King $H$, et al. Understanding variation in rates of referral among general practitioners: are inappropriate referrals important and would guidelines help to reduce rates? BMJ 1993;307:1467-70.

13. Harbour R, Miller J. for the Scottish Intercollegiate Guidelines Network Grading Review Group. A new system for grading recommendations in evidence based guidelines. BMJ 2001;323:334-6.

14. NHS centre for reviews and dissemination. Undertaking systematic reviews of research on effectiveness: CRD's guidance for those carrying out or commissioning reviews, CRD report number 4 2nd edn. March 2001. York, UK: University of York.

15. Thomas R, Grimshaw J, Mollison J, et al. Cluster randomized trial of a guidelinebased open access urological investigation service. Fam Pract 2003;20:646-54.

16. Morrison J, Carroll L, Twaddle S, et al. Pragmatic randomised controlled trial to evaluate guidelines for the management of infertility across the primary care-secondary care interface. BMJ 2001;322:1282-4.

17. Goldberg H, Deyo R, Taylor $\mathrm{V}$, et al. Can evidence change the rate of back surgery? A randomized trial of community-based education. Eff Clin Pract 2001;4:95-104.

18. Fender G, Prentice A, Gorst $\mathrm{T}$, et al. Randomised controlled trial of educational package on management of menorrhagia in primary care: the Anglia menorrhagia evaluation study. BMJ 1999:318:1246-50.

19. Emslie C, Grimshaw J, Templeton A. Do clinical guidelines improve general practice management and referral of infertile couples? BMJ 1993;306:1728-31.

20. Maddison P, Jones J, Breslin A, et al. Improved access and targeting of musculoskeletal services in northwest Wales: targeted early access to musculoskeletal services (TEAMS) programme. BMJ 2004;329:1325-7.

21. Benninger $\mathbf{M}$, King $\mathbf{F}$, Nichols R. Management guidelines for improvement of otolaryngology referrals from primary care physicians. Otolaryngol Head Neck Surg 1995; 113:446-52.

22. Rao J, Kroenke K, Mihaliak K, et al. Can guidelines impact the ordering of magnetic resonance imaging studies by primary care providers for low back pain? Am J Manag Care 2002;8:27-35

23. Spatafora S, Canepa G, Migliari R, et al. Effects of a shared protocol between urologists and general practitioners on referral patterns and initial diagnostic 
management of men with lower urinary tract symptoms in Italy: the Prostate Destination study. BJU Int 2005;95:563-70.

24. Julian S, Naftalin N, Clark M, et al. An integrated care pathway for menorrhagia across the primary-secondary interface: patients' experience, clinical outcomes, and service utilisation. Qual Saf Health Care 2007;16:110-15

25. Bradshaw C, McColl E, Pritchett $\mathrm{C}$, et al. The south Tyneside FASTRAK service: evaluation of a new model for day surgery. Ambul Surg 1997:5:171-7.

26. Kumar B, Walsh R, Courteney-Harris R, et al. Direct listing' for tonsillectomy by general practitioners. Clin Otolaryngol Allied Sci 1998;23:91-2.

27. Collins $\mathbf{M}$, Barry $M$, Bin $\mathrm{L}$, et al. Diagnosis and treatment of benign prostatic hyperplasia. Practice patterns of primary care physicians. J Gen Intern Med 1997; 12:224-9.

28. Friedlieb $\mathbf{0}$. The impact of managed care on the diagnosis and treatment of low back pain: a preliminary report. Am J Med Oual 1994:9:24-9.

29. Rossignol M, Abenhaim L, Bonvalot $Y$, et al. Should the gap be filled between guidelines and actual practice for management of low back pain in primary care? The Quebec experience. Spine 1996;21:2893-8.

30. Bishop $\mathbf{P}$, Wing P. Compliance with clinical practice guidelines in family physicians managing worker's compensation board patients with acute lower back pain. Spine J $2003 \cdot 3 \cdot 442-50$
31. Cerdan Carbonero M, Sanz L, Martinez R. Improving communication between levels of health care: direct referral of patients to a one-stop service for major outpatient surgery. Aten Primaria 2005;35:283-7.

32. Arroyo A, Andreu J, Garcia P, et al. [Analysis of a programme of direct referral between primary and specialist care in potential surgery patients]. Aten Primaria 2001:28:381-5.

33. Padilla L, Marchal E, Caballero A, et al. [Shared care in BPH. First national experience]. Actas Urol Esp. 1998;22:478-83.

34. Norg R, Portjegis P, van de Beek K, et al. A decision aid for GPs for the treatment of elderly male patients with lower urinary tract symptoms (LUTS) Fam Pract. 2006;23:666-73

35. Rao C. Halsey J, Bukhart M, et al. Referrals to hospital based rheumatology and orthopaedic services: seeking direction. Rheumatology 2005:44:1332.

36. Lash S. Referrals for cataract and action on cataracts, evidence-based guidelines. Br J Gen Pract. 2005 December 1:55:965-6.

37. Fullen B, Maher T, Bury G, et al. Adherence of Irish general practitioners to European guidelines for acute low back pain: a prospective pilot study. Eur J Pain 2007;11:614-23.

38. Mays N, Pope C, Popay J. Systematically reviewing qualitative and quantitative evidence to inform management and policy-making in the health field. $J$ Health Serv. Res Pol. 2005:S1:6-20.

\section{Guidelines International Network Conference 2010}

August 25 - 28, 2010

Chicago, Illinois USA

\section{Registration Now Open}

\section{Join your colleagues at the G-I-N Conference 2010 to:}

- Bridge the disciplines of evidence-based medicine, translational research, quality improvement, and continuing medical education to drive best practices.
- Promote the scientific development, dissemination, implementation, and evaluation of guidelines to advance health care.

- Learn how to foster evidencebased guidelines as the foundation for improved quality in patient care.

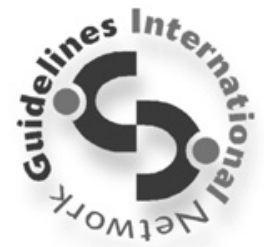

Last Chance to Register and Save

Register by June 24 - Save up to $\$ 220$ www.gin2010.org
Attend presentations by international leaders and experts in health-care research and policy.

- Take home new knowledge and ideas to enhance your efforts and facilitate improvements in medical practice.

\section{INTEGRATING KNOWLEDGE. IMPROVING OUTCOMES.}

